This item was submitted to Loughborough's Research Repository by the author.

Items in Figshare are protected by copyright, with all rights reserved, unless otherwise indicated.

\title{
Fault detection and isolation filter design for systems subject to polytopic
} uncertainties

\section{PLEASE CITE THE PUBLISHED VERSION}

\section{PUBLISHER}

(C) IEEE

\section{VERSION}

VoR (Version of Record)

\section{LICENCE}

CC BY-NC-ND 4.0

\section{REPOSITORY RECORD}

Mazars, E., I.M. Jaimoukha, Zhenhai Li, and Argyrios C. Zolotas. 2019. "Fault Detection and Isolation Filter Design for Systems Subject to Polytopic Uncertainties". figshare. https://hdl.handle.net/2134/4300. 
This item was submitted to Loughborough's Institutional Repository (https://dspace.lboro.ac.uk/) by the author and is made available under the following Creative Commons Licence conditions.

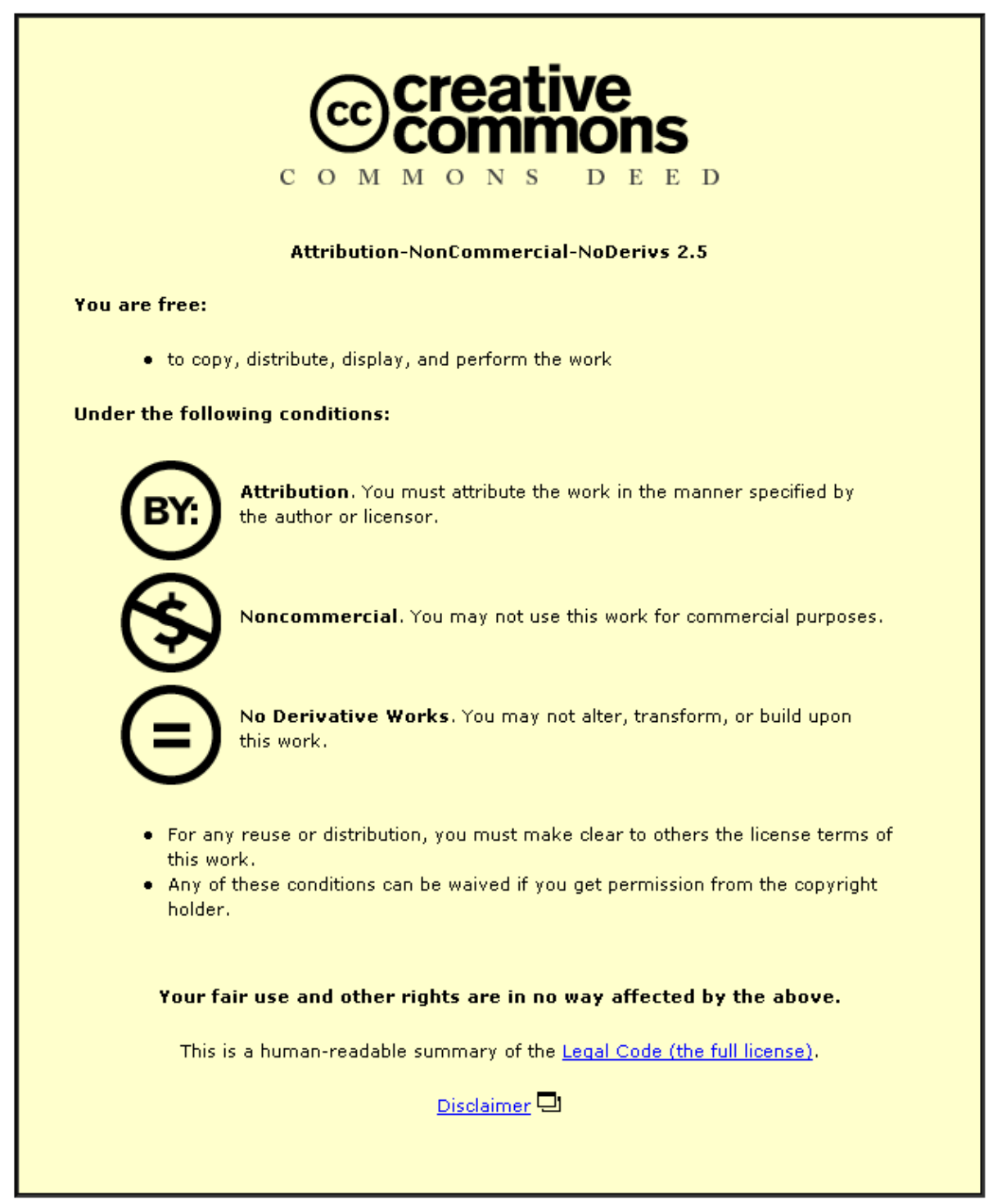

For the full text of this licence, please go to: http://creativecommons.org/licenses/by-nc-nd/2.5/ 


\title{
Fault Detection and Isolation Filter Design for Systems subject to Polytopic Uncertainties
}

\author{
E. Mazars*, I.M. Jaimoukha*, Z. Li*, A. C. Zolotas ${ }^{\dagger}$ \\ ${ }^{*}$ Control and Power Group, Department of Electrical and Electronic Engineering, \\ Imperial College, London, U.K. \\ ${ }^{\dagger}$ Department of Electronic and Electrical Engineering, Loughborough University, U.K.
}

\begin{abstract}
This paper considers the robust fault detection and isolation (FDI) problem for linear time-invariant dynamic systems subject to faults, disturbances and polytopic uncertainties. We employ an observer-based FDI filter to generate a residual signal. We propose a cost function that penalizes a weighted combination of the deviation of the fault to residual dynamics from a given fault isolation reference model, as well as the effects of disturbances and uncertainties on the residual, using the $\mathcal{H}_{\infty}$ norm as a measure. The proposed cost function thus captures the requirements of fault detection and isolation and disturbance rejection in the presence of polytopic uncertainties. We derive necessary and sufficient conditions for the existence of an FDI filter that achieves the design specifications. This condition takes the form of easily implementable linear matrix inequality (LMI) optimization problem.
\end{abstract}

\section{INTRODUCTION}

As science and technology develops, the reliability and security of complex systems becomes more important. In recent years, Model-based FDI schemes exploiting analytic redundancy have received increasing attention in the literature and applications [1]. The schemes involve the design of an observer which provides a fault indicating signal (residual) using input and output signals from the monitored system. However, there is always a model reality mismatch between plant dynamics and the model used for the residual generation. The residual is sensitive to faults, disturbances and plant/model mismatch. The filter design objective is then to reduce the sensitivity to disturbances and plant/model mismatch as well as isolating faults. The performance of an FDI system should therefore be measured by a suitable trade off between robustness and sensitivity.

In the exact fault detection approach (see [2], [3]) the requirement is that the residual signal is decoupled from disturbances, while the transfer function matrix from faults to residual has a specific, typically diagonal, structure for fault detection and isolation. Almost disturbance decoupling (see [4]) requires that the transfer function matrix from the disturbance to the residual signal be arbitrarily small in either the $\mathcal{H}_{2}$ or $\mathcal{H}_{\infty}$ norm sense rather than requiring it to be exactly zero. The weakening of the exact disturbance decoupling requirement to an almost disturbance decoupling requirement makes great sense from an engineering point of view. Other approaches include a matrix pencil approach [5], and a matrix factorization approach [6].

Recently developed LMI approaches have received much attention for formulating FDI decoupling problems
[7], [8]. Hou and Patton gave a realization of fault detection observer design based on the bounded real lemma [9]. Casavola et al. worked on a novel solution to robust FDI for linear polytopic uncertain plants using deconvolution filters [10]. Zhong et al. proposed a new performance index by introducing a reference residual model, formulated using LMI techniques [11]. Using these techniques, the decoupling problem can be transformed to a sensitivity optimization problem, which seeks to increase the sensitivity of the residual to faults and simultaneously reduce the sensitivity to disturbances and plant/model mismatch. However, isolation is employed indirectly in the above methods through the use of banks of observers. This makes it hard to deal with multiple faults (where faults might occur simultaneously). Furthermore, these approaches recast model uncertainties as system disturbances, which restricts the class of model uncertainties that can be handled.

The robust FDI problem is closely related to the robust $\mathcal{H}_{\infty}$ filtering, which estimates the states of a system using the past measurements. The $\mathcal{H}_{\infty}$-filter is designed such that the $\mathcal{H}_{\infty}$-norm of the system, which reflects the worst case "gain" of the system, is minimized (see [12]). Recently, LMI techniques were recently considered by Sun and Packard [13], to design a filter robust to both structured and unstructured norm-bounded uncertainties.

In this work, an FDI filter is constructed such that the $\mathcal{H}_{\infty}$-norm of the transfer matrix function from both disturbances and plant/model mismatch to the residual is minimized, with the constraint the transfer matrix function from faults to the residual is close to a diagonal transfer matrix function (called reference model). The filter can provide robustness to uncertainties in all system matrices. Sufficient and necessary conditions for the existence of such a filter and the optimal solution are given via solving LMIs.

This paper is organized as follows. After defining the notation, we review filter-based fault detection and isolation techniques for residual signal generation and give the problem formulation in Section II. Section III presents a linear matrix inequality formulation for the FDI problem and gives the optimal solution. Finally, a numerical example is presented in Section IV and Section V summarizes our results.

The notation we use is fairly standard. The set of real (complex) $n \times m$ matrices is denoted by $\mathcal{R}^{n \times m}\left(\mathcal{C}^{n \times m}\right)$. For $A \in \mathcal{C}^{n \times m}$ we use the notation $A^{\prime}$ to denote the 
complex conjugate transpose. A matrix $A \in \mathcal{C}^{n \times n}$ is called Hermitian if $A=A^{\prime}$. For a Hermitian matrix $A \in \mathcal{C}^{n \times n}, A \succ 0(A \prec 0)$ denotes that $A$ is positive (negative) definite, that is, all the eigenvalues of $A$ are greater (less) than zero. The $n \times n$ identity matrix is denoted as $I_{n}$ and the $n \times m$ null matrix is denoted as $0_{n, m}$ with the subscripts occasionally dropped if they can be inferred from context.

$\mathcal{R}(s)^{m \times p}$ denotes the set of all $m \times p$ proper, realrational matrix functions of $s . \mathcal{L}_{\infty}^{m \times p}$ denotes the space of $m \times p$ matrix functions with entries bounded on the extended imaginary axis. The subspace $\mathcal{H}_{\infty}^{m \times p} \subset \mathcal{L}_{\infty}^{m \times p}$ denotes matrix functions analytic in the closed right-half of the complex plane. A prefix $\mathcal{R}$ denotes a real-rational function, so that $\mathcal{R} \mathcal{H}_{\infty}^{m \times p}$ denotes the set of all $m \times p$ stable real-rational matrix functions of $s$. Let $\mathcal{L}^{2}$ be the set of functions that are square integrable, the $\mathcal{L}^{2}$-norm of a function $u \in \mathcal{L}^{2}$ is defined as:

$$
\|u\|_{2}=\left(\int_{0}^{+\infty}|u(t)|^{2} d t\right)^{\frac{1}{2}} .
$$

For $G(s) \in \mathcal{R} \mathcal{H}_{\infty}^{m \times p}$ we define

$$
\|G\|_{\infty}=\sup _{\substack{u \in \mathcal{L}^{2} \\ u \neq 0}} \frac{\|G u\|_{2}}{\|u\|_{2}} .
$$

\section{PROBLEM FORMULATION}

Let us consider the following set of polytopic uncertainties:

$$
\left.\begin{array}{rl}
\Omega & =\left\{\left[\begin{array}{llll}
A(\xi) & B(\xi) & B_{d}(\xi) & B_{f}(\xi) \\
C(\xi) & D(\xi) & D_{d}(\xi) & D_{f}(\xi)
\end{array}\right]\right. \\
& =\sum_{i=1}^{p} \xi_{i}\left[\begin{array}{llll}
A_{i} & B_{i} & B_{d, i} & B_{f, i} \\
C_{i} & D_{i} & D_{d, i} & D_{f, i}
\end{array}\right], \sum_{i=1}^{p} \xi_{i}=1, \quad \xi_{i} \geq 0
\end{array}\right\}
$$

where $A_{i}, B_{i}, C_{i}, D_{i}, B_{d, i}, B_{f, i}, D_{d, i}$ and $D_{f, i}(i=$ $1, . ., p)$ are known constant matrices with appropriate dimensions, and $\xi_{i}(i=1, . ., p)$ are time-invariant uncertainties.

We therefore consider the following system, subject to disturbances, modeling errors and process, sensor and actuator faults modeled as

$\left\{\dot{x}(t)=A(\xi) x(t)+B(\xi) u(t)+B_{d}(\xi) d(t)+B_{f}(\xi) f(t)\right.$, $\left\{y(t)=C(\xi) x(t)+D(\xi) u(t)+D_{d}(\xi) d(t)+D_{f}(\xi) f(t)\right.$,

where $\left[\begin{array}{rrrr}A(\xi) & B(\xi) & B_{d}(\xi) & B_{f}(\xi) \\ C(\xi) & D(\xi) & D_{d}(\xi) & D_{f}(\xi)\end{array}\right] \in \Omega, x(t) \in$ $\mathcal{R}^{n}, u(t) \in \mathcal{R}^{n_{u}}$ and $y(t) \in \mathcal{R}^{n_{y}}$ are the process state, input and output vectors, respectively, and where $d(t) \in \mathcal{R}^{n_{d}}$ and $f(t) \in \mathcal{R}^{n_{f}}$ are the disturbance and fault vectors, respectively. Here, $B_{f}(\xi) \in \mathcal{R}^{n \times n_{f}}$ and $D_{f}(\xi) \in \mathcal{R}^{n_{y} \times n_{f}}$ are the component and instrument fault distribution matrices, respectively, while $B_{d}(\xi) \in \mathcal{R}^{n \times n_{d}}$ and $D_{d}(\xi) \in \mathcal{R}^{n_{y} \times n_{d}}$ are the corresponding disturbance distribution matrices [14]. Henceforth, we use $A$ to denote $A(\xi)$, similarly for $B, C, D, B_{d}, B_{f}, D_{d}$ and $D_{f}$.

In general, a residual signal in an FDI system should represent the inconsistency between the actual system variables and the mathematical model, and respond to faults, disturbances and modeling errors only.

The objective is to design a fault detection and isolation filter of the form

$$
\left\{\begin{array}{l}
\dot{\hat{x}}(t)=A_{k} \hat{x}(t)+B_{k u} u(t)+B_{k y} y(t), \\
\left.r(t)=C_{k} \hat{x}(t)+D_{k u} u(t)+D_{k y} y(t)\right),
\end{array}\right.
$$

where $\hat{x}(t) \in \mathcal{R}^{n_{k}}$ is the filter state and $r(t) \in \mathcal{R}^{n_{f}}$ is the residual signal. Here $A_{k}, C_{k}, B_{k u}, B_{k y}, D_{k u}, D_{k y}$ are to be determined. Figure 1 shows this filter in the robust residual generation scheme.

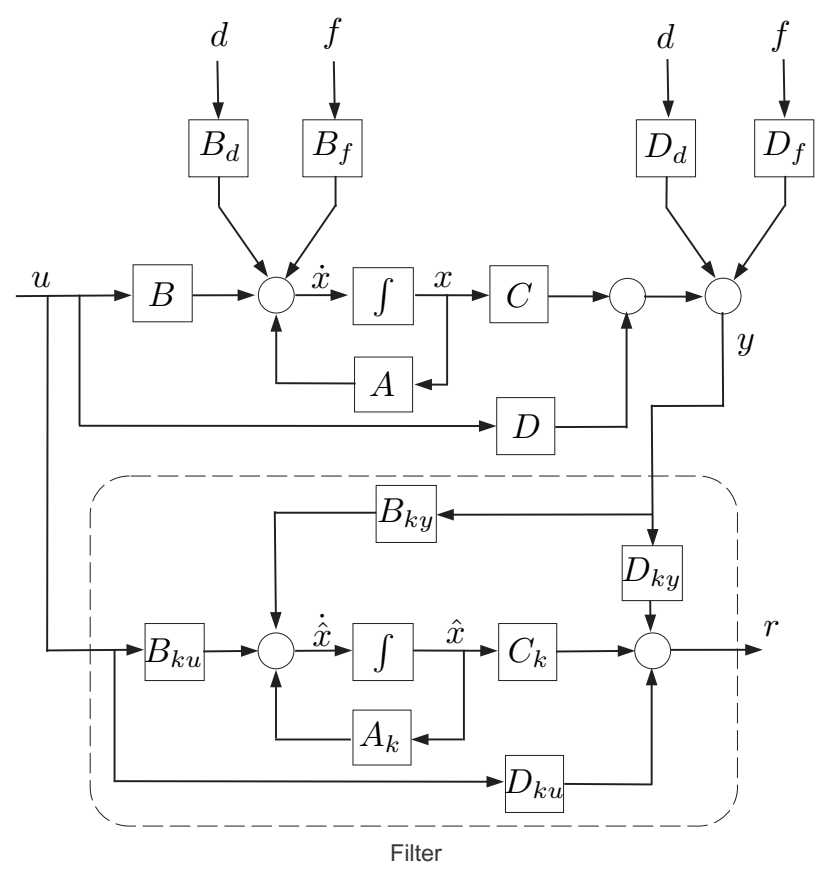

Fig. 1. Filter-based robust FDI scheme

We use the filter described in (1). We get the following expression for the residual:

$$
r(s)=T_{r f}(s) f(s)+T_{r d}(s) d(s)+T_{r u}(s) u(s),
$$

where

$\left[\begin{array}{lll}T_{r f} & T_{r d} & T_{r u}\end{array}\right] \stackrel{s}{=}\left[\begin{array}{l|lll}\tilde{A} & \tilde{B}_{f} & \tilde{B}_{d} & \tilde{B}_{u} \\ \hline \tilde{C} & \tilde{D}_{f} & \tilde{D}_{d} & \tilde{D}_{u}\end{array}\right]$

$$
\stackrel{s}{=}\left[\begin{array}{cc|ccc}
A & 0 & B_{f} & B_{d} & B \\
B_{k y} C & A_{k} & B_{k y} D_{f} & B_{k y} D_{d} & B_{k u}+B_{k y} D \\
\hline D_{k y} C & C_{k} & D_{k y} D_{f} & D_{k y} D_{d} & D_{k y} D
\end{array}\right]
$$

are the transfer matrices from faults, disturbances and inputs to the residual, respectively. Note that the dynamics of the residual signal depend not only on $f$ and $d$ but also on $u$ and $x$.

For exact fault detection, the residual signal is required to be sensitive only to faults. This is equivalent to the requirement that $T_{r d}=0, T_{r u}=0$ and $T_{r f} \neq 0$. For exact fault isolation, it is further required that the fault signature be deduced from the residual. This is equivalent to the requirement that $T_{r d}=0, T_{r u}=0$ and $T_{r f} \in$ $\Sigma$, where $\Sigma$ is a set of transfer matrices with a special structure that depends on the nature of the faults. For example, if all the faults can occur simultaneously, then $\Sigma$ 
is the set of stable diagonal transfer matrices with nonzero diagonal entries. Due to the modelling uncertainties, exact fault detection and isolation is not possible.

For robust FDI, we propose the following, more realistic, problem formulation.

Problem 2.1: With all variables as defined above, assume that the original system dynamics are quadratically stable and let

$$
T_{o} \stackrel{s}{=}\left[\begin{array}{l|l}
A_{o} & B_{o} \\
\hline C_{o} & D_{o}
\end{array}\right]
$$

denote a given stable fault reference dynamics, where $A_{o} \in \mathcal{R}^{n_{o} \times n_{o}}, B_{o} \in \mathcal{R}^{n_{o} \times n_{f}}, C_{o} \in \mathcal{R}^{n_{f} \times n_{o}}$ and $D_{o} \in \mathcal{R}^{n_{f} \times n_{f}}$, and let $\alpha \in[0,1]$ be given. For any $\gamma>0$, find a stable filter of the form given in (1), if it exists, such that

$$
\sup _{M \in M_{\xi}}\left\|\left[\alpha\left(T_{r f}-T_{o}\right) \quad \beta T_{r d} \quad \beta T_{r u}\right]\right\|_{\infty}<\gamma,
$$

where $\beta=1-\alpha$. It is clear that reducing $\left\|T_{r f}-T_{o}\right\|_{\infty}$ will improve fault isolation and that reducing $\left\|T_{r d}\right\|_{\infty}^{\infty}$ and $\left\|T_{r u}\right\|_{\infty}$ will improve fault detection. By choosing a suitable $\alpha$, we can emphasize fault detection or fault isolation since optimizing one will generally affect the performance of the other. In the sequel, we assume that $\alpha$ and $\beta$ are absorbed in the system data.

Among the model-based approaches, the most common way is the observer-based approach (Chen and Patton [15], Lien [16]), which uses state observers to generate residuals. Here we do not consider an observer but a more general filter that is widely used in other concepts. We slightly modified this filter by adding the matrix $B_{k u}$ so as to handle the robust FDI problem.

In the case of exact decoupling without uncertainties, a common assumption in the fault detection problem is that $D_{f}=0$ and/or $C B_{f}$ has full rank [17], [18], [19]. While in the almost decoupling approach, the assumption $D_{f}$ has full rank is widely used [6], [20]. Here we do not impose any of these assumptions.

\section{LINEAR MATRIX INEQUALITY FORMULATION}

We consider in this section a matrix inequality formulation approach. The main idea is to express the inequality (2) in a linear matrix inequality formulation using the bounded real lemma, then deriving sufficient and necessary conditions for solvability.

We consider that the reference model $T_{o}$ is known and therefore set to a given value, which may be chosen using engineering criteria that depend on the nature of the faults. See [21], [11] for more details on the choice of $T_{o}$.

The cost function in (2) can be written as

$$
\begin{gathered}
{\left[\begin{array}{ccc}
T_{r f}-T_{o} & T_{r d} & T_{r u}
\end{array}\right] \stackrel{s}{=}\left[\begin{array}{c|c|c}
A_{c} & B_{c} \\
\hline C_{c} & D_{c}
\end{array}\right]} \\
:=\left[\begin{array}{cc|ccc}
A_{o} & 0 & B_{o} & 0 & 0 \\
0 & \tilde{A} & \tilde{B}_{f} & \tilde{B}_{d} & \tilde{B}_{u} \\
\hline-C_{o} & \tilde{C} & \tilde{D}_{f}-D_{o} & \tilde{D}_{d} & \tilde{D}_{u}
\end{array}\right] .
\end{gathered}
$$

It follows from the bounded real lemma that (2) is satisfied if and only if there exists $P_{c}=P_{c}^{\prime} \in$
$\mathcal{R}^{\left(n_{o}+n+n_{k}\right) \times\left(n_{o}+n+n_{k}\right)}$ such that $P_{c} \succ 0$ and

$$
\left[\begin{array}{ccc}
A_{c}^{\prime} P_{c}+P_{c} A_{c} & P_{c} B_{c} & C_{c}^{\prime} \\
B_{c}^{\prime} P_{c} & -\gamma^{2} I & D_{c}^{\prime} \\
C_{c} & D_{c} & -I
\end{array}\right] \prec 0
$$

(See Theorem 3 in Tuan et al [22]). Now

$$
\left[\begin{array}{c|c}
A_{c} & B_{c} \\
\hline C_{c} & D_{c}
\end{array}\right] \in\left\{\sum_{i=1}^{p} \xi_{i}\left[\begin{array}{c|c}
A_{c}^{i} & B_{c}^{i} \\
\hline C_{c}^{i} & D_{c}^{i}
\end{array}\right], \quad \sum_{i=1}^{p} \xi_{i}=1, \quad \xi_{i} \geq 0\right\}
$$

where

$\left[\begin{array}{c|c}A_{c}^{i} & B_{c}^{i} \\ \hline C_{c}^{i} & D_{c}^{i}\end{array}\right]=$

$\left[\begin{array}{ccc|ccc}A_{o} & 0 & 0 & B_{o} & 0 & 0 \\ 0 & A_{i} & 0 & B_{f, i} & B_{d, i} & B_{i} \\ 0 & B_{k y} C_{i} & A_{k} & B_{k y} D_{f, i} & B_{k y} D_{d, i} & B_{k u}+B_{k y} D_{i} \\ \hline-C_{o} & D_{k y} C_{i} & C_{k} & D_{k y} D_{f, i}-D_{o} & D_{k y} D_{d, i} & D_{k u}+D_{k y} D_{i}\end{array}\right]$

We first assume that the polytopic system is quadratically stable (See Gahinet et al [23] and Boyd et al[24]). Recall that (2) is satisfied if and only if (3) is satisfied (Tuan et al [22]). Now

$$
\begin{gathered}
(3) \Leftrightarrow\left\{\sum_{i=1}^{p} \xi_{i}\left[\begin{array}{ccc}
A_{c}^{i^{\prime}} P_{c}+P_{c} A_{c}^{i} & P_{c} B_{c}^{i} & C_{c}^{i^{\prime}} \\
B_{c}^{i^{\prime}} P_{c} & -\gamma^{2} I & D_{c}^{i^{\prime}} \\
C_{c}^{i} & D_{c}^{i} & -I
\end{array}\right] \prec 0,\right. \\
\left.\forall \xi_{i} \text { s.t. } \sum_{i=1}^{p} \xi_{i}=1, \xi_{i} \geq 0\right\} \\
\left.\Leftrightarrow\{\underbrace{\left[\begin{array}{ccc}
A_{c}^{i^{\prime}} P_{c}+P_{c} A_{c}^{i} & P_{c} B_{c}^{i} & C_{c}^{i^{\prime}} \\
B_{c}^{i^{\prime}} P_{c} & -\gamma^{2} I & D_{c}^{i^{\prime}} \\
C_{c}^{i} & D_{c}^{i} & -I
\end{array}\right]}_{T_{p o l}^{i}}\} 0, \forall i=1, . . p\right\}
\end{gathered}
$$

Since the above matrix inequality is nonlinear, we use a change of variable to turn it into LMIs (Scherer et al.[25]). Assume that $n_{k}=n_{o}+n$, that is, the order of the filter is equal to the order of the system dynamics plus the order of the fault reference model. Partition $P_{c}$ and $P_{c}^{-1}$ as

$$
P_{c}=\left[\begin{array}{cc}
Y & N \\
N^{\prime} & \hat{Y}
\end{array}\right], \quad P_{c}^{-1}=\left[\begin{array}{cc}
X & M \\
M^{\prime} & \hat{X}
\end{array}\right]
$$

where $X, Y, \hat{X}, \hat{Y} \in \mathcal{R}^{n_{k} \times n_{k}}$ are symmetric. From $P_{c} P_{c}^{-1}=I$, we infer $P_{c}\left[\begin{array}{c}X \\ M^{\prime}\end{array}\right]=\left[\begin{array}{l}I \\ 0\end{array}\right]$ which leads to

$P_{c} \Pi_{1}=\Pi_{2} \quad$ with $\quad \Pi_{1}=\left[\begin{array}{cc}I & I \\ M^{\prime} Z & 0\end{array}\right], \quad \Pi_{2}=\left[\begin{array}{cc}Z & Y \\ 0 & N^{\prime}\end{array}\right]$

where $Z=X^{-1}$.

Define $T=\operatorname{diag}\left(\Pi_{1}, I\right)$. Then $T_{\text {pol }}^{i} \prec 0 \Leftrightarrow \bar{T}_{\text {pol }}^{i}:=$ $T T_{\text {pol }}^{i} T^{\prime} \prec 0$. This results in the following calculations, where boldface letters are used to emphasize the LMI 
optimization variables:

$$
\begin{aligned}
& \Lambda_{11}^{i}:=\Pi_{1}^{\prime} P_{c} A_{c}^{i} \Pi_{1} \\
& =\left[\begin{array}{cc}
\boldsymbol{Z} \bar{A}^{i} & \boldsymbol{Z} \bar{A}^{i} \\
\boldsymbol{Y} \bar{A}^{i}+\hat{\boldsymbol{A}}+\hat{\boldsymbol{B}}_{\boldsymbol{y}} \bar{C}^{i} & \boldsymbol{Y} \bar{A}^{i}+\hat{\boldsymbol{B}}_{\boldsymbol{y}} \bar{C}^{i}
\end{array}\right] \\
& \Lambda_{12}^{i}:=\left(\Pi_{1}^{\prime} P_{c} B_{c}^{i}\right)^{\prime} \\
& =\left[\begin{array}{cc}
\left(\boldsymbol{Z} \bar{B}_{f}^{i}\right)^{\prime} & \left(\boldsymbol{Y} \bar{B}_{f}^{i}+\hat{\boldsymbol{B}}_{\boldsymbol{y}} D_{f}^{i}\right)^{\prime} \\
\left(\boldsymbol{Z} \bar{B}_{d}^{i}\right)^{\prime} & \left(\boldsymbol{Y} \bar{B}_{d}^{i}+\hat{\boldsymbol{B}}_{\boldsymbol{y}} D_{d}^{i}\right)^{\prime} \\
\left(\boldsymbol{Z} \bar{B}^{i}\right)^{\prime} & \left(\boldsymbol{Y} \bar{B}^{i}+\hat{\boldsymbol{B}}_{\boldsymbol{y}} D^{i}+\hat{\boldsymbol{B}}_{\boldsymbol{u}}\right)^{\prime}
\end{array}\right]^{\prime} \\
& \Lambda_{13}^{i}:=\left(C_{c}^{i} \Pi_{1}\right)^{\prime} \\
& =\left[\begin{array}{ll}
\hat{\boldsymbol{D}}_{\boldsymbol{y}} \bar{C}^{i}-\bar{C}_{o}+\hat{\boldsymbol{C}} & \hat{\boldsymbol{D}}_{\boldsymbol{y}} \bar{C}^{i}-\bar{C}_{o}
\end{array}\right]^{\prime} \\
& \Lambda_{23}^{i}=\left[\begin{array}{c}
D_{f}^{i^{\prime}} \hat{\boldsymbol{D}}_{\boldsymbol{y}}{ }^{\prime}-D_{o}^{\prime} \\
D_{d}^{i^{\prime}} \hat{\boldsymbol{D}}_{\boldsymbol{y}}{ }^{\prime} \\
D^{i^{\prime}} \hat{\boldsymbol{D}}_{\boldsymbol{y}}{ }^{\prime}+\hat{\boldsymbol{D}}_{\boldsymbol{u}}{ }^{\prime}
\end{array}\right],
\end{aligned}
$$

where we have defined

$$
\begin{aligned}
{\left[\begin{array}{lll}
\bar{A}^{i} \bar{B}_{f}^{i} & \bar{B}_{d}^{i} \bar{B}^{i}
\end{array}\right] } & :=\left[\begin{array}{cc|ccc}
A_{o} & 0 & B_{o} & 0 & 0 \\
0 & A^{i} & B_{f}^{i} & B_{d}^{i} & B^{i}
\end{array}\right] \\
{\left[\begin{array}{c}
\bar{C}^{i} \\
\bar{C}_{o}
\end{array}\right] } & :=\left[\begin{array}{cc}
0 & C^{i} \\
C_{o} & 0
\end{array}\right]
\end{aligned}
$$

and the new variables

$$
\left[\begin{array}{ccc}
\hat{A} & \hat{B}_{y} & \hat{B}_{u} \\
\hat{C} & \hat{D}_{y} & \hat{D}_{u}
\end{array}\right]=\left[\begin{array}{ccc}
N A_{k} M^{\prime} Z & N B_{k y} & N B_{k u} \\
C_{k} M^{\prime} Z & D_{k y} & D_{k u}
\end{array}\right]
$$

If $\boldsymbol{M}$ and $\boldsymbol{N}$ are invertible, the variable $\boldsymbol{A}_{k}, \boldsymbol{C}_{k}, \boldsymbol{B}_{k y}$, $\boldsymbol{B}_{k u}, \boldsymbol{D}_{k y}, \boldsymbol{D}_{k u}$ can be replaced by $\hat{A}, \hat{\boldsymbol{B}}_{\boldsymbol{y}}, \hat{\boldsymbol{B}}_{\boldsymbol{u}}, \hat{\boldsymbol{C}}, \hat{\boldsymbol{D}}_{\boldsymbol{y}}$, $\hat{D}_{u}$ without loss of generality.

We can now rewrite $\bar{T}_{\text {pol }}^{i}$ with the new variables, we get

$$
\bar{T}_{p o l}^{i}=\left[\begin{array}{ccc}
\Lambda_{11}^{i}+\Lambda_{11}^{i^{\prime}} & \Lambda_{12}^{i} & \Lambda_{13}^{i} \\
* & -\gamma^{2} I & \Lambda_{23}^{i} \\
* & * & -I
\end{array}\right] \prec 0,
$$

which is linear in the variables $\Lambda_{i j}$ 's that are defined in (4)-(7). Regarding the constraint $P_{c} \succ 0$, it is straightforward to express it as an LMI in $\boldsymbol{Y}$ and $\boldsymbol{Z}$ :

$$
P_{c} \succ 0 \Leftrightarrow \Pi_{1}^{\prime} P_{c} \Pi_{1} \succ 0 \Leftrightarrow\left[\begin{array}{cc}
\boldsymbol{Z} & \boldsymbol{Z} \\
\boldsymbol{Z} & \boldsymbol{Y}
\end{array}\right] \succ 0 .
$$

Therefore we can derive a sufficient and necessary condition for solvability of Problem 2.1 in a form of a linear matrix inequality formulation as follows:

Lemma 3.1: Suppose that the polytopic system is quadratically stable. Then (2) is satisfied if there exist $\hat{A}, \hat{B}_{y}, \hat{B}_{u}, \hat{C}, \hat{D}_{y}, \hat{D}_{u}$ and symmetric matrices $\boldsymbol{Y}$ and $Z$ such that (8) and

$$
\bar{T}_{p o l}^{i} \prec 0 \quad \forall i=1, . ., p
$$

are satisfied.

\section{NUMERICAL EXAMPLE}

To illustrate the effectiveness of the proposed fault detection and isolation filter scheme, a numerical example is considered in this section. A randomly generated example is given as

$$
\begin{aligned}
& {\left[\begin{array}{ll}
A_{1} & A_{2}
\end{array}\right]=\left[\begin{array}{cc||cc}
-1.2046 & 0.6952 & -2.6155 & 2.8933 \\
-0.1632 & -0.7501 & 0.6806 & -2.2006
\end{array}\right],} \\
& {\left[\begin{array}{ll}
B_{1} & B_{2}
\end{array}\right]=\left[\begin{array}{cc||cc}
0.1893 & 0.3689 & -0.0930 & 1.1238 \\
-0.6660 & -0.1792 & 0.6038 & 0.4739
\end{array}\right],} \\
& {\left[\begin{array}{ll}
C_{1} & C_{2}
\end{array}\right]=\left[\begin{array}{cc||cc}
0.8589 & -0.4968 & -0.1705 & 0.8243 \\
1.5757 & 0.9342 & 0.5726 & 0.2175 \\
1.7888 & 0.8670 & 0.4826 & -0.7804
\end{array}\right],} \\
& {\left[\begin{array}{ll}
D_{1} & D_{2}
\end{array}\right]=\left[\begin{array}{ll||ll}
0.2632 & 0.6371 & 0.8021 & 0.8206 \\
0.7138 & 0.5459 & 0.6683 & 0.9705 \\
0.9776 & 0.8481 & 0.6710 & 0.4869
\end{array}\right]}
\end{aligned}
$$

This system is subject to three disturbances and two potential faults. Here, the setup is given by

$$
\begin{aligned}
& B_{d, 1}=\left[\begin{array}{ccc}
0.1963 & 0.0853 & 0.1420 \\
0.0861 & -0.0106 & 0.2293
\end{array}\right], \\
& B_{d, 2}=\left[\begin{array}{ccc}
-0.2548 & -0.0121 & 0.0633 \\
0.0849 & 0.0281 & 0.0064
\end{array}\right] \text {, } \\
& B_{f, 1}=\left[\begin{array}{cc}
-1.3892 & 0.9012 \\
-0.6455 & -1.0858
\end{array}\right] \text {, } \\
& B_{f, 2}=\left[\begin{array}{cc}
-0.9280 & 1.0008 \\
0.9126 & -1.0194
\end{array}\right] \text {, } \\
& D_{d, 1}=\left[\begin{array}{ccc}
-0.1333 & -0.0552 & -0.0698 \\
-0.1373 & 0.0474 & -0.0857 \\
0.0842 & -0.0601 & -0.1130
\end{array}\right] \text {, } \\
& D_{d, 2}=\left[\begin{array}{ccc}
0.0773 & -0.0623 & -0.1330 \\
-0.0289 & 0.0093 & -0.0375 \\
0.0993 & -0.0078 & -0.0837
\end{array}\right] \text {, } \\
& D_{f, 1}=\left[\begin{array}{cc}
-0.3441 & 1.1247 \\
-0.3710 & -1.0590 \\
-1.5788 & 0.7079
\end{array}\right] \\
& D_{f, 2}=\left[\begin{array}{cc}
-2.1251 & 1.6963 \\
-0.8484 & 0.7223 \\
-2.0174 & -1.5747
\end{array}\right] \text {. }
\end{aligned}
$$

We implemented the algorithm in Matlab to minimize $\gamma$, and we have chosen the cost functions $W_{f}, W_{d}$ and $W_{u}$ to be identity as a compromised between fault detection and fault isolation. Simulated through MATLAB and SIMULINK, these disturbances are two band limited white noises with mean 0 and standard deviation 1 , and a positive jump from the $5^{\text {th }}$ second. Fault $f_{1}$ and $f_{2}$, simulated by a unit positive and negative jump respectively, are connected from the $14^{t h}$ and $22^{t h}$ second respectively. The input $u$ is taken as a periodic signal. We eventually get:

$$
\gamma=1.2487
$$

Figure 2 gives the residual responses, where each fault can be readily distinguished from the others and the disturbances. It is worth noting that, in order to verify the effectiveness of the optimal filter, similar amplitudes for the disturbance and faults are assumed, which is quite demanding from the practical point of view.

This example makes clear that the designed filter satisfies the performance requirement of rapid detection and 


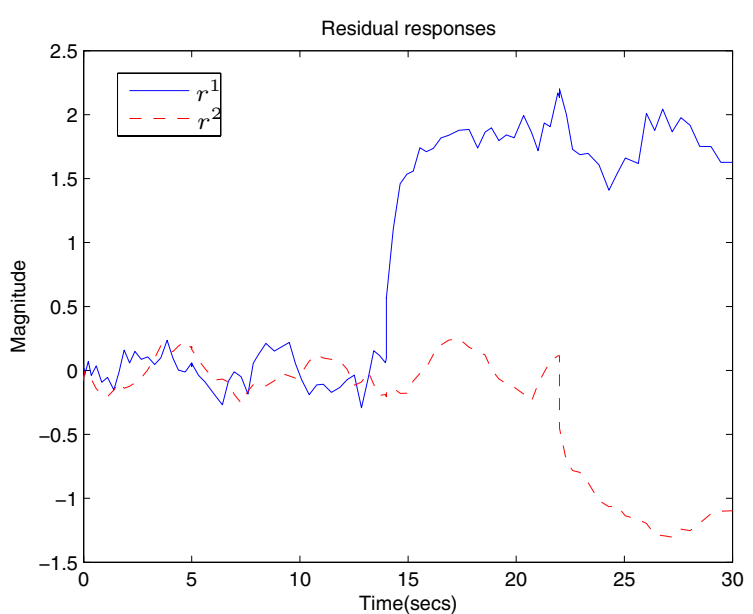

Fig. 2. Time response of the residual

direct fault isolation which is sufficiently robust against disturbances and modeling errors.

\section{CONCLUSION}

In this paper, we have considered a robust fault detection and isolation scheme for systems which are affected by polytopic uncertainties, using a matrix inequality formulation approach. We construct a filter that bounds the influence of the disturbances and model uncertainties on the residual signal measured in terms of the $\mathcal{H}_{\infty}-$ norm. We give a sufficient and necessary condition for solvability, as well as the optimal solution of the robust FDI problem in a form of LMIs. Furthermore, our scheme implies that each element of the residual is only sensitive to a specified potential fault and therefore can handle multiple faults (where faults might occur at the same time). It is worth noting that our scheme can also be applied when model uncertainties are not considered. A numerical example demonstrates the effectiveness of the proposed approach.

\section{REFERENCES}

[1] Z. Gao and H. Wang, "Descriptor observer approaches for multivariable systems with measurement noises and application in fault detection and diagnosis," Systems and Control Letters, vol. 55, no. 4, pp. 304-313, 2006.

[2] R. Patton and J. Chen, "On eigenstructure assignment for robust fault diagnosis," Int. J. Robust \& Nonlinear Control, vol. 10, no. 14, pp. 1193-1208, 2000.

[3] P. Zhang, H. Ye, S. Ding, G. Wang, and D. Zhou, "On the relationship between parity space and $\langle\in$ approaches to fault detection," System and Control Lettres, vol. 55, pp. 94-100, 2006.

[4] M. Sadrnia, J. Chen, and R. Patton, "Robust fault diagnosis observer design using $\mathcal{H}_{\infty}$ optimisation and $\mu$ synthesis," IEE Colloquium on Modelling and Signal Processing for Fault Diagnosis, pp. 9/1-16, 1996.

[5] R. Patton and M. Hou, "Design of fault detection and isolation observers: a matrix pencil approach," Automatica, vol. 34, no. 9, pp. 1135-1140, 1998.

[6] I. Jaimoukha, Z. Li, and V. Papakos, "A matrix factorization to the $\mathcal{H}_{-} / \mathcal{H}_{\infty}$ fault detection problem," Automatica, vol. 42 , no. 11 , pp. 1907-1912, 2006.

[7] I. Jaimoukha, Z. Li, and E. Mazars, "Fault isolation filter with linear matrix inequality solution to optimal decoupling," in Proc. Amer. Control Conf., Minneapolis, Minnesota, USA, June 2006.
[8] D. Henry and A. Zolghadri, "Design of fault diagnosis filters: A multi-objective approach," Journal of the Franklin Institute, vol. 34, pp. 421-446, 2005.

[9] M. Hou and R. Patton, "An LMI approach to $\mathcal{H}_{-} / \mathcal{H}_{\infty}$ fault detection observers," in UKACC International Conference on Control. '96, England, 1996, pp. 305-310.

[10] A. Casavola, D. Famularo, and G. Franze, "A robust deconvolution scheme for fault detection and isolation of uncertain linear systems: an lmi approach," Automatica, vol. 41, no. 8, pp. 1463 1472,2005

[11] M. Zhong, S. Ding, J. Lam, and H. Wang, "An LMI approach to design robust fault detection filter for uncertain LTI systems," Automatica, vol. 39, no. 3, pp. 543-550, 2003.

[12] K. M. Nagpal and P. P. Khargonekar, "Filtering and smoothing in an $\mathcal{H}_{\infty}$ setting," IEEE Trans. Automatic Control, vol. 36, no. 2, pp. 152-166, 1991.

[13] K. Sun and A. Packard, "Robust $\mathcal{H}_{2}$ and $\mathcal{H}_{\infty}$ filters for uncertain lft systems," IEEE Trans. Automatic Control, vol. 50, no. 5, pp. 715-720, 2005.

[14] P. Frank and X. Ding, "Survey of robust residual generation and evaluation methods in observer-based fault detection systems," $J$. Proc. Cont., vol. 7, no. 6, pp. 403-424, 1997.

[15] J. Chen and P. Patton, Robust model-based fault diagnosis for dynamic systems. Boston: Kluwer Academic Publishers, 1999.

[16] C. Lien, "An efficient method to design robust observer- based control of uncertain linear systems," Applied Mathematics and Computation, vol. 158, no. 1, pp. 29-44, 2004.

[17] W. H. Chung and J. L. Speyer, "A game theoretic fault detection filter," IEEE Trans. Automatic Control, vol. 43, no. 2, pp. 143-161, 1998.

[18] R. Douglas and J. Speyer, "Robust fault detection filter design," in Proc. Amer. Control Conf., Seattle, Washington, June 1995, pp. 91-96, IEEE Press, New York.

[19] J. Park, G. Rizzoni, and W. Ribbens, "On the representation of sensor faults in fault detection filters," Automatica, vol. 30, no. 11, pp. 1793-1795, 1994.

[20] H. Wang, L. Lam, S. Ding, and M. Zhong, "Iterative linear matrix inequality algorithms for fault detection with unknown inputs," Journal of Systems and Control Engineering, vol. 219, no. 2, pp. 161-172, August 2005

[21] E. Frisk and L. Nielsen, "Robust residual generation for diagnosis including a reference model for residual behavior," in Proceedings of the 14th IFAC World Congress, Beijing, China, 1999, pp. 55-60.

[22] H. Tuan, P. Apkarian, and T. Nguyen, "Robust filtering for uncertain nonlinearly parametrized plants," IEEE Transsaction on Signal Processing, vol. 51, no. 7, pp. 1806-1815, 2003.

[23] P. Gahinet, P. Apkarian, and Chilali, "parameter dependent lyapunov functions for real parametric uncertainties," IEEE Trans. Automatic Control, vol. 41, pp. 436-442, 1996.

[24] S. Boyd, L. El Ghaoui, E. Feron, and V. Balakrishnan, Linear Matrix Inequalities in Systems and Control Theory. Philadelphia, PA: SIAM Publications, 1994.

[25] C. Scherer, P. Gahinet, and M. Chilali, "Multi-objective outputfeedback control via LMI optimization," IEEE Trans. Automatic Control, vol. 42, no. 7, pp. 896-911, 1997. 